\title{
Interdisziplinäre Aufgabe Diabetiker mit Herzschwäche: Sanft zum Zucker, hart gegen Lipide!
}

- Diabetiker mit Herzinsuffizienz benötigen eine strikte LDL-Cholesterin-Senkung sowie eine moderate Blutzuckereinstellung auf 7-8\%, bei der Hypoglykämien unbedingt zu vermeiden sind. Darauf machte Prof. Carsten Tschöpe, Charité Berlin, beim Diabetes-Weltkongress aufmerksam.

Diabetiker sterben heute überwiegend an kardiovaskulären Erkrankungen, 40\% entwickeln eine Herzinsuffizienz, wobei die Inzidenz ab dem Alter von 55 Jahren stark steigt. Sie erkranken häufiger als Nichtdiabetiker an systolischer (SH) und diastolischer Herzinsuffizienz (DH). Allerdings ist die DH nicht leicht zu diagnostizieren: Es kommt zu Belastungsdyspnoe, aber Pumpfunktion und Ventrikelgröße liegen noch im Normbereich. Um zu erkennen, wann der Diabetes das Herz erreicht, sollte einmal jährlich eine Gewebe-Doppler-Echokardiografie erfolgen, die eine diastolische Dysfunktion aufdecken kann, so Tschöpe. Im Zweifel kann ein Stressecho sinnvoll sein.

Wenn eine DH bei einem Diabetiker diagnostiziert ist, hat dies zwei Konsequenzen: Zum einen muss eine Hochdrucktherapie von einer bei Diabetes idealen Kombination aus ACE-Hemmer oder $\mathrm{AT}_{1}$-Blocker plus Kalziumantagonist - etwa Valsartan/Amlodipin (Exforge ${ }^{\circ}$ - gegebenenfalls durch ein Diuretikum ergänzt werden. Sollte sich im Verlauf eine SH mit reduzierter Ejektionsfraktion eingestellt haben, ist der Kalziumantagonist abzusetzen, so Tschöpe. Der Grund ist die negative Inotropie der Arznei.

Zum anderen ist die Herzinsuffizienz des Diabetikers stark mit Vorhofflimmern assoziiert, das die Prognose weiter verschlechtert. Daher ist ein jährliches Langzeit-EKG zu fordern, falls entsprechende Risikofaktoren vorliegen wie große Vorhöfe.

Bei Typ-2-Diabetes LDL-Ziel generell $<70$ mg/dl In der üblichen kardiologischen Therapie ist die Lipidsenkung die prognostisch wichtigste Maßnahme, so Tschöpe. Hier geben die neuen europäischen Leitlinien nun striktere Zielwerte vor: Bei allen Typ-2sowie bei allen Typ-1-Diabetikern mit Endorganschäden wird heute ein LDL-Ziel von $70 \mathrm{mg} / \mathrm{dl}$ vorgegeben. Dieses ist, so Tschöpe, oft nur in Kombitherapien (Statin + Ezetimib oder Statin + retardierte Nikotinsäure) zu erreichen. Alternativ sollte das Ausgangs-LDL mindestens um 50\% gesenkt werden.

Für den Blutdruck gilt ein Ziel von 130-140 mmHg systolisch, wobei $130 \mathrm{mmHg}$ nicht unterschritten werden sollten, sofern der Patient eine KHK hat. de

Quelle: Experten-Workshop Welt-Diabetes-Kongress IDF in Dubai, Dezember 2011, Veranstalter: Novartis

\section{Einstieg in die Insulintherapie Effektiv und flexibel mit Basalinsulin-Analogon}

- Für den Einstieg in die Insulintherapie bei Typ-2Diabetes wird meist ein Basalinsulin gewählt, im weiteren Verlauf ist dann oft ein prandiales Insulin zusätzlich nötig. Ein solches Konzept ist flexibler und effektiver als die Gabe eines Intermediärinsulins.

„Die mit einem Basalinsulin unterstützte orale Therapie hat sich als einfach und effektiv etabliert", sagte Prof. Reinhard G. Bretzel, Gießen. Die Wirksamkeit des Basalinsulin-Analogons Insulinglargin (Lantus $^{\circledast}$ ) in dieser Kombination zur Blutzuckeroptimierung sei hinlänglich dokumentiert. Wenn damit kein optimaler postprandialer Blutzucker erreichbar ist, sei zusätzlich ein kurz wirksames Insulin nötig.

Eine aktuelle Metaanalyse mit 12 Studien ergab, dass die frühe Gabe von Glargin zusätzlich zu Metformin vorteilhafter ist als Glargin plus Sulfonylharnstoff $(\mathrm{SH})$. „Dies gilt besonders in einem frühen Diabetesstadium“, so Bretzel. Dann könne mit der Kombination Glargin-Metformin eine stärkere $\mathrm{HbA}_{1 \mathrm{c}}$-Reduktion bei geringerem Hypoglykämierisko erreicht werden als in Kombination mit SH. In einer 60-wöchigen Open-Label-Studie wurden drei inten- sivierte Insulinregime zusätzlich zu oralen Antidiabetika (OAD) bei nicht ausreichend eingestellten Typ-2-Diabetikern verglichen. Ein Drittel der Patienten erhielt Glargin plus maximal eine Injektion des kurz wirksamen Insulinanalogons Glulisin (Apidra ${ }^{\circ}$ ) zur Mahlzeit, ein Drittel Glargin plus bis zu drei Injektionen Glulisin, und ein Drittel ein Mischinsulin zweimal täglich. Glargin plus einmal Glulisin sei dem Mischinsulin überlegen gewesen, so Bretzel. Nicht nur die Effektivität, auch die Flexibilität spreche für das kurz wirksame Insulinanalogon.

Daten, die beim EASD-Kongress 2011 in Lissabon vorgestellt wurden, zeigen zudem, dass der Therapieeinstieg mit Glargin bei ähnlich geringer Gewichtszunahme zu einer besseren Blutzuckereinstellung führt als andere Insuline, OAD oder Ernährungsumstellungen. „Die geringste Gewichtszunahme wurde bei einem Ausgangs- $\mathrm{HbA}_{1 \mathrm{c}}$ von $<8 \%$ sowie bei über 65-Jährigen beobachtet“, sagte Bretzel. sti

Quelle: Pressekonferenz, Jahrestagung Stiftung „Der herzkranke Diabetiker" 2.12.2011 in Berlin. Veranstalter: Sanofi 